\title{
ZUSAMMENFASSUNGEN RÉSUMÉS
}

Die folgenden Texte geben eine kurze Inhaltszusammenfassung der französischsprachigen Beiträge in Deutsch und der deutschsprachigen Texte in Französisch. Sie sind als sprachliche Verständnishilfe gedacht. Aus thematischen Gründen entspricht die Reihenfolge der Anordnung der Aufsätze innerhalb des Bandes.

Les textes suivants livrent de brefs résumés du contenu des contributions en langue allemande en français et des textes français en allemand. Ils sont destinés à faciliter la compréhension linguistique. Pour des raisons thématiques, l'ordre retenu correspond à celui des articles dans ce volume.

\section{Gisela Naegle, Einleitung}

En France, en Bourgogne, dans l'Empire médiéval, en Espagne (Castille et Catalogne) et dans des villes italiennes comme Florence, le maintien de la paix et la question de la défense étaient intimement liés. Pour sauvegarder la paix d'une manière durable, il fallait garantir la sécurité et organiser une défense efficace. Depuis quelques décennies, le maintien de la paix, les négociations diplomatiques et les discours sur la paix, le règlement judiciaire et extrajudiciaire des conflits, les vengeances, »guerres privées«/faides, Fehden, ligues urbaines, Landfriedensbünde et luttes de factions à l'intérieur des villes ont fait l'objet de nombreuses études. $\mathrm{Au}$ cours des conflits et des procédures de pacification, plusieurs facteurs étaient susceptibles de jouer rôle décisif. L'impact du concept de l'honneur et les valeurs sociales des acteurs politiques offrent des perspectives intéressantes de comparaison. Existe-il des différences importantes entre les conflits urbains et nobiliaires ou bien peut-on constater une influence prépondérante du modèle aristocratique? Quel est le modèle du règlement de conflits entre >partenaires` d'origines sociales et géographiques très différentes? La comparaison des résultats de ces études récentes - qui résultent parfois d'approches méthodiques très diverses et fortement influencées par les traditions historiographiques et les disciplines scientifiques respectives - figurait parmi les objectifs du colloque. Dans le cadre de la thématique générale, une attention particulière a été accordée au dialogue scientifique franco-allemand et aux recherches publiées en langues romanes.

Le point de départ des réflexions qui suivent est la question posée par Marc Bloch dans un discours devant le Congrès international des sciences historiques de 1928. En répondant à la question »Qu'est-ce, tout d'abord, dans notre domaine, que comparer? «, il parlait de la nécessité d'intégrer et d'admettre non seulement les similitudes, mais également les différences. Ce constat n'a rien perdu de son actualité.

Présentant un grand nombre de liens d'>interaction< directs, les régions choisies pour terrain d'enquête offrent de très riches points d'ancrage à une étude comparative. Des conflits tels que la guerre de Cent Ans ou la guerre civile catalane eurent des répercussions sur l'équilibre politique européen et contribuèrent au développement de 
l'art de la diplomatie et des >techniques` de la négociation. La terminologie des sources latines et des langues vernaculaires, les œuvres d'auteurs de traités théoriques et juridiques répandus, les conciles en tant que »foires d'idées" et forums de discussion à l'échelle européenne fournirent partout des supports d'idées et des occasions d'alimenter la réflexion médiévale sur la paix, la guerre et la défense. Dans toutes les régions mentionnées, la réception plus ou moins profonde du droit savant romanocanonique et ses relations avec les coutumes locales jouèrent un rôle important dans l'histoire de la réflexion sur la paix. Des adages juridiques comme vim vi repellere licet ou la citation du décret de Gratien Non pax queritur, ut bellum exerceatur, sed bellum geritur, ut pax acquiratur (Decretum Grat. C. 23 q. 2 c. 3 ) influencèrent et nourrirent un débat théorique qui n'était pas sujet aux frontières politiques de l'époque. Les relations avec les gens d'armes, les anciens soldats et mercenaires déracinés de la guerre de Cent Ans, la violence des nobles, la faide (Fehde), les crimes de guerre et la recherche de modes effectifs de sauvegarde de la paix constituèrent des préoccupations communes provoquant une évolution des formes de coopération plus ou moins institutionnalisées entre les villes, les membres de la noblesse et du clergé, les états, les princes et la royauté. Dans plusieurs cas, on peut constater la mise en place de systèmes collectifs de défense sous la forme de ligues urbaines ou nobiliaires, comme les ligues de paix territoriales (Landfriedensbünde) dans l'Empire ou les hermandades en Espagne.

\section{Jean-Marie Moeglin, À la recherche de la "paix finale»}

[Hundertjähriger Krieg, Frankreich/England, 14. Jh.]

Am Anfang der Untersuchung steht die Feststellung, die Geschichte des Hundertjährigen Krieges sei häufig als Kriegsgeschichte geschrieben worden, obwohl es eigentlich zutreffender wäre, sie als Geschichte der Suche nach einem endgültigen Frieden zu schreiben. Als einer Suche, die zwar von einer gewissen Anzahl militärischer Episoden unterbrochen wurde, die aber letztlich hauptsächlich das Ziel verfolgt habe, den Gegner zu Friedensverhandlungen zu zwingen. Dennoch sei dieser ersehnte Frieden letztlich unerreichbar gewesen, auch wenn man zweimal, in Troyes (1420) und in Brétigny (1360), glauben konnte, endlich am Ziel zu sein.

In chronologischer Hinsicht konzentriert sich der stark auf die Quellenüberlieferung der englischen Seite gestützte Beitrag auf die Beziehungen zwischen dem englischen und dem französischen König in der ersten Hälfte des Hundertjährigen Krieges. Dabei lassen sich dem Autor zufolge fünf Modelle von Beziehungen zwischen Herrschern und Staaten unterscheiden, die einerseits einem chronologischen Ablauf gefolgt, andererseits aber auch zeitlich synchron miteinander aufgetreten seien:

- Die Interpretation des Verhältnisses der beiden Könige als Vassalitätsbeziehung des englischen Königs als Herzog von Aquitanien zu seinem französischen Gegner, - der Anspruch des englischen Königs auf die französische Krone als ritterliches >Unternehmen< (emprise chevaleresque),

- Krieg und Frieden als Vereinbarung zwischen beiden Königreichen,

- der endgültige Frieden als Vorbedingung der Eintracht zwischen beiden Königen und ihren Königreichen mit dem Ziel eines gemeinsamen Vorgehens gegen die »Ungläubigen«,

- der Frieden durch ein Ehebündnis zwischen den Herrscherhäusern beider Länder (Heirat Richards II. mit der Tochter Charles VI., 1396). 


\title{
Christine Reinle, Legitimation und Delegitimierung von Fehden
}

\author{
[Empire médiéval, bas Moyen Âge]
}

Selon l'hypothèse initiale de l'auteur, le fait que les monarchies de l'Europe de l'Ouest étaient à de nombreuses reprises confrontées à des ennemis extérieurs puissants serait à l'origine d'une différence importante avec la situation observée dans l'Empire médiéval. Dans ce dernier, les tentatives de limiter l'autodéfense armée, et particulièrement celle des nobles, n'auraient guère connu de succès. Les mesures contre les excès de la faide (Fehde) y rencontrèrent des obstacles considérables. L'empereur fut fréquemment impliqué de manière active dans des querelles régionales de faible périmètre qui l'opposèrent aux princes territoriaux et autres détenteurs locaux du pouvoir. Ainsi la distinction entre conflits »privés« et »publics« et la terminologie du droit savant instruisant une opposition entre "guerre privée « et "guerre publique« ne sauraient-elles être appliquées à la réalité constitutionnelle de l'Empire.

L'étude examine ensuite le règlement de la Fehde dans les sources normatives telles que le droit féodal du »Miroir des Saxons« (Sachsenspiegel), les sentences des échevins de Magdebourg (Magdeburger Schöppensprüche), le Weistum d'Oppenheim de 1419, ainsi que dans les traités théoriques et les œuvres d'auteurs tels que Heinrich von Gorkum († 1431, compositeur du traité »De bello iusto«), Heinrich von Langenstein $(† 1397)$, Stephan von Landskron $(† 1477)$ et Bruder Berthold. L'une des thèses centrales tourne autour des conséquences de la réception >populaire nique par le biais de la catéchèse et des sommes de confesseurs. Destinés à un public élargi, ces œuvres et les consilia des juristes de l'époque auraient joué un rôle décisif dans la propagation des idées savantes sur la condamnation de la violence et de la Fehde. Ceci vaudrait particulièrement dans le milieu nobiliaire, mais également dans les différentes couches de la société. Jusqu'à présent, la recherche a plutôt négligé cet aspect, et l'auteur invite à approfondir l'étude de la réception et de la diffusion de ces œuvres. En revanche, en dehors des milieux érudits des canonistes, la réception des concepts du droit romano-canonique aurait été relativement tardive et hésitante dans l’Empire médiéval.

\section{Horst Carl, Landfrieden als Konzept und Realität kollektiver Sicherheit}

$$
\text { [Empire médiéval, } \mathrm{XV}^{\mathrm{e}}-\mathrm{XVI}^{\mathrm{e}} \text { siècle] }
$$

La communication porte sur le concept et la réalité des paix territoriales (Landfrieden) en tant que systèmes de défense collective dans l'Empire médiéval et au début des Temps modernes. L'auteur souligne la liaison étroite entre la structure fédérale de l'Empire et le développement spécifique de l'>instrument des paix territoriales. Les ligues de paix territoriales (Landfriedenseinungen) avaient pour fonction de >compenser< les carences et espaces vides entre les petits territoires et les nombreux seigneurs concurrents. Selon lui, les transitions entre les associations nobiliaires (adelige Einungen) et les différentes formes de représentation collective à l'échelle des états régionaux (landständische Repräsentation) étaient floues. Contrairement à l'opinion défendue par Otto von Gierke, l'importance du modèle constitutionnel de la Einung devrait être quelque peu relativisée puisqu'il ne s'agirait pas là d'une spécificité de l'évolution médiévale de l'Empire. De même, ce modèle ne devrait pas être considéré comme la forme constitutionnelle centrale des structures politiques de l'Empire de l'époque. L'étude des associations nobiliaires montrerait que des formes d'organisation >horizontales` de caractère égalitaire (genossenschaftliche Organisationsformen) et des structures hiérarchiques d'ordre ne seraient pas inconciliables ou incom- 
patibles. Le fonctionnement concret d'un tel système collectif pour la sauvegarde de la paix est illustré par l'exemple de la ligue de Souabe (Schwäbischer Bund, 14881534). Dans la dernière phase de son existence, cette ligue regroupa tous les territoires importants de l'Allemagne du Sud, ainsi que des territoires de moindre importance: parmi ses membres figuraient des princes, le roi, des villes et des nobles. Son but était de freiner l'exercice du droit du plus fort et de proposer des procédures de règlement des conflits. La ligue de Souabe connut des succès militaires tangibles et faisait office d'arbitre. Loin de constituer un contre-modèle, les tentatives de réforme de l'Empire proclamées au cours de la diète de Worms en 1495, parmi lesquelles on compte la Paix perpétuelle (Ewiger Landfrieden), profitèrent des expériences acquises par la ligue de Souabe. Pour le $\mathrm{XVI}^{\mathrm{e}}$ siècle, l'historiographie s'est surtout focalisée sur l'action de la ligue contre les paysans révoltés (1525). Néanmoins, par le biais de l'instrument juridique du Landfriedensrecht, c'est-à-dire des normes juridiques issues des paix territoriales, cette ligue tenta également avec succès de trouver une solution aux conflits religieux naissants. Son arbitrage restait attractif, car elle s'efforçait de séparer strictement les questions du maintien de la paix territoriale de celles de la >vérité` théologique religieuse.

\section{Máximo Diago Hernando, Die politische Rolle der Städtebünde}

\section{[Castille, $\mathrm{XIII}^{\mathrm{e}}-\mathrm{XVI}^{\mathrm{e}}$ siècle]}

La Castille, l'Italie du Nord et l'Empire médiéval connurent une période intensive de développement de ligues urbaines. Contrairement à l'Italie et l'Allemagne, dès le $\mathrm{XIII}^{\mathrm{e}}$ siècle, la Castille fut caractérisée par un pouvoir monarchique fort qui montra une tendance prononcée à la centralisation. En Castille, le roi fut considéré comme le principal responsable et garant du maintien de la paix. Néanmoins, plusieurs périodes de crise du pouvoir et de faiblesse de la royauté furent propices à la constitution de ligues régionales et générales de villes. En 1282, le conflit entre le roi Alphonse X (1221-1284) et son héritier, le futur roi Sancho IV, donna lieu à la constitution d'une Hermandad General. Les minorités des rois Ferdinand IV (1285-1312) et Alphonse XI (1311-1350) provoquèrent des luttes de factions. Ainsi, le dernier quart du $\mathrm{XIII}^{\mathrm{e}}$ siècle et le premier quart du XIV furent l'apogée du développement de hermandades interrégionales et d'un rôle politique très actif des villes. Au sein des hermandades, les villes exercèrent une fonction stabilisatrice en faveur du pouvoir royal. Mais les ligues urbaines agirent également à titre d'instrument de défense de l'autonomie de leurs membres et du système juridique des fueros contre l'introduction du Fuero Real et les aspirations centralisatrices de la monarchie. Selon la démonstration de l'auteur, en dépit de nombreuses différences, on peut observer des parallèles entre ces phénomènes et la constitution de la Ligue rhénane de 1254, car dans les deux cas les crises du pouvoir royal favorisèrent l'activité et le développement de ligues et d'autres formes associatives de défense collective des intérêts des villes et des nobles, ainsi qu'une participation politique accrue des villes. Cependant, les intérêts des villes et de la noblesse furent parfois divergents. Entre le XIII ${ }^{\mathrm{e}}$ et le XVI $\mathrm{X}^{\mathrm{e}}$ siècle, les hermandades connurent une transformation profonde. Dans ce contexte, le processus d'aristocratisation des élites dirigeantes des villes joua un rôle important, propre à accentuer les différences en comparaison avec l'évolution des ligues urbaines allemandes. À la fin de la période étudiée, la royauté parvint à transformer l'institution des hermandades en un instrument au service de ses propres intérêts et à l'utiliser aux fins de la conquista du royaume de Grenade. Créée par les Rois Catholiques et dotée de fonctions de police, la Hermandad General devint un instrument du maintien de la 
paix et de la lutte contre la criminalité dans les régions rurales. Au cours de l'insurrection des Comuneros, sous le règne du roi Charles I ${ }^{\mathrm{er}}$ (l'empereur Charles Quint), le modèle des hermandades dans leur sens originel faisait encore office de point de référence.

\section{Stéphane Péquignot, "La pràticha de aquesta ciutat e principat»}

[Barcelona/Katalonien, 1461-1464]

In den Jahren zwischen 1460 und 1472 kam es zu einem heftigen Konflikt zwischen König Johann (Juan) II. von Aragón (1458-1479) und den wichtigsten katalanischen Institutionen. Dabei gingen die ungelösten Probleme im Verhältnis der Katalanen zu ihrem neuen Herrscher bereits auf die Regierungszeit seines Vorgängers Alfons des Großmütigen (Alfonso el Magnánimo, 1416-1458) zurück. Hinzu kamen Spannungen zwischen Johann II. und seinem Sohn Karl von Viana (Carlos de Viana), der von den Katalanen gegen den Vater unterstützt wurde und dessen Tod ein empfindliches Gleichgewicht ins Wanken brachte. Im weiteren Verlauf der Ereignisse erklärten die katalanischen Machthaber den König, die Königin und ihren Sohn Ferdinand aufgrund der Verletzung des Vertrags von Vilafranca del Penedès zu Feinden. Diese Situation veranlasste rasch eine Vielzahl weiterer Akteure zum Eingreifen, zumal sich die katalanische Seite aktiv um Unterstützung von außen bemühte. Zu den sauswärtigen Akteuren des Konfliktes gehörten in dieser Zeit sowohl der französische König Louis XI. als auch König Heinrich (Enrique) IV. von Kastilien (1462-1464), der Infant Peter (Pedro) von Portugal (1464-1466) und René von Anjou (1466-1472). Der katalanische Bürgerkrieg gab in den vergangenen Jahrzehnten Anlass zu einer Reihe unterschiedlicher Bewertungen und stößt in den letzten Jahren auf ein zunehmendes Interesse der Forschung. Im Zentrum des Beitrags stehen die Beziehungen der katalanischen Seite zu den rausländischen Akteuren in der Zeit zwischen Oktober 1461 und Januar 1464, dem Ende der Herrschaft Heinrichs IV. von Kastilien. In dieser Zeit kam es zu intensiven diplomatischen Aktivitäten, Verhandlungen und zahlreichen Gesandtschaften in mehrere europäische Länder und zur römischen Kurie. Die Analyse bezieht sich auf Sprache, Argumentationsmuster und Diskurse der Verhandlungen sowie auf die im Titel erwähnte »diplomatische Praxis«. Dabei erwies sich vor allem die in diplomatischen Angelegenheiten erfahrene Stadt Barcelona als zentrale >Drehscheibe< der Verhandlungen. In der Vergangenheit aufgetretene Spannungen zwischen den verschiedenen katalanischen Autoritäten zwangen jedoch auch zu einem ständigen internen Interessenausgleich. Um einen stabilen Frieden zu erreichen, war es schließlich notwendig, sich einem Herrn zu unterstellen, da die Katalanen innerhalb des europäischen Kräftespiels sonst allenfalls mit Stadtstaaten auf gleicher Ebene verhandeln konnten.

\section{Christiane Klapisch-Zuber, Le prince et la paix des familles à Florence}

[Florenz, 14. Jh.]

Am Anfang der Untersuchung steht die Feststellung, Frieden sei weniger ein zu erreichendes Ziel als eine Modalität der Rückkehr zu einem Gleichgewicht der Ehre. Im 13. und 14. Jahrhundert sei das öffentliche Leben in einer italienischen Großstadt wie Florenz ganz durch den Wettstreit zwischen Familien dominiert und durch Parteikämpfe determiniert gewesen. Den Hintergrund dieser Konflikte bildete zunächst der 
Gegensatz zwischen Guelfen und Gibellinen, danach die Auseinandersetzung zwischen popularen Großen (grands populaires, ital. popolani) und Magnaten (magnats, ital. magnati). In der ersten Hälfte des 14. Jahrhunderts kamen während der Regierungszeit des der Anjou-Dynastie angehörenden Königs Robert von Sizilien-Neapel (1278-1343) und der Kirche durch sog. pacieri in ca. 90 Fällen Friedenschlüsse zustande. Der König präsentierte sich auf diese Weise als idealer Garant der Legitimierung und Durchsetzung des städtischen Friedens.

Im Mittelpunkt der Ausführungen steht die Zeit des 1343 als >Tyrann aus Florenz vertriebenen Gautier de Brienne, Herzog von Athen (1304/5-1356). In den Jahren 1342-1343 kamen unter seiner Herrschaft 266 aus einem Notariatsregister bekannte Friedensschlüsse zustande. Die Auswertung dieser Quellen und die Herkunft der beteiligten Parteien zeigen, dass es sich nicht um ein rein aristokratisches Modell der Konfliktregelung handelte. Es gab ein Kontinuum und fließende Übergänge zwischen von vor Notaren registrierten Friedensschlüssen und den unter kommunaler oder herzoglicher Autorität beschworenen bzw. erzwungenen Frieden. Eine strenge Unterscheidung zwischen »öffentlich" und "privat" ist nach Ansicht der Autorin daher in diesem Fall ohne heuristisches Interesse, da in Florenz der politische Sektor und die Definition des Gemeinwohls durch die von den Familien vorgegebenen Strukturen bestimmt gewesen seien.

\section{Julie Claustre, Assurer la paix des ménages}

$$
\text { [Paris, 14.-15. Jh.] }
$$

Der Beitrag beschäftigt sich anhand der Problematik schuldrechtlicher Konflikte mit der Frage, ob es unabhängig von der Natur der jeweiligen Auseinandersetzungen (um Sachen, Personen oder Ehre) eine gemeinsame »Grammatik« mittelalterlicher Konfliktlösungsformen gab, die in unterschiedlichen sozialen Segmenten und sowohl auf der kollektiven als auch auf der individuellen Ebene Anwendung fand. Als »Testfall« werden kleinteilige schuldrechtliche Konflikte gewählt, die in der Regel auf einige Haushalte beschränkt blieben. Schulden und Kreditbeziehungen waren im spätmittelalterlichen Europa sehr weit verbreitete Phänomene, wobei neben halbprofessionellen Kreditformen, wie Vereinbarungen unter Kaufleuten, auch Hilfeleistungen im Rahmen der Solidarität zwischen Verwandten und Nachbarn, zwischen Grundherren und Bauern usw. eine wichtige Rolle spielten. Die gewaltsame Eskalation von Schuldverhältnissen bis hin zu Rachemaßnahmen und Tötungsdelikten, aber auch die gerichtliche bzw. außergerichtliche Befriedung solcher Auseinandersetzungen werden anhand von mehreren Fallbeispielen aus dem spätmittelalterlichen Paris analysiert. In diesem Zusammenhang spielten der Rückgriff auf die Gerichtsbarkeit und Zwangsmaßnahmen wie die Schuldhaft durchaus eine ambivalente Rolle. Sie konnten sowohl durch die öffentliche Stigmatisierung von Betroffenen zur Verschärfung von Konflikten führen und als »Rache« des Gläubigers gedeutet werden, als auch eine Wiederaufnahme von Verhandlungen zwischen den gegnerischen Parteien bewirken. Diese verhandlungsfördernde Funktion der Schuldhaft zeigt sich besonders an ihrer kurzen Dauer, da die Hälfte der Inhaftierten weniger als 48 Stunden in Haft blieb. Im Zusammenhang mit der Regelung von Schuldrechtskonflikten kam es zu einer zunehmenden Verschriftlichung und Notare spielten eine wichtige Rolle. Das angemessene Verhalten von Schuldnern und Gläubigern war darüber hinaus Gegenstand theoretischer und literarischer Werke mit didaktischen Intentionen. 


\section{Bertrand Schnerb, Sauver les meubles}

[Normandie, Anfang 15. Jh.]

Die Kapitulation von Festungen und Städten ist ein Phänomen, das sich besonders gut zu Beginn des 15. Jahrhunderts beobachten lässt. Dies gilt vor allem für die vom englischen König Henry V. (1366-1413) in der Normandie geführte Feldzugskampagne. Henry V. benutzte damals Verhandlungen als zusätzliches taktisches Mittel und Ergänzung der militärischen Eroberung. In den Jahren 1417 bis 1419 wurden über vierzig Kapitulationsverträge geschlossen. Dieses Textkorpus, das bisher nur sehr punktuell für Monographien oder zu chronologischen Zwecken herangezogen wurde, erlaubt auch einen methodischen Zugriff, der den Ablauf der Kapitulation, die Verhandlungen und die jeweiligen Zielsetzungen der Parteien in den Vordergrund stellt. Im Zentrum der Überlegungen steht daher die Reflexion über das Verhältnis und die Wechselwirkungen von militärischer Aktion und Diplomatie, von Krieg und Frieden und die Frage des Ausgleichs zwischen den Erfordernissen der Verteidigung und des Schutzes individueller Interessen. Zu den Untersuchungsgegenständen gehört auch die Typologie der Verhandlungskommissionen, die sich in Abhängigkeit von der Größe und Bedeutung der betroffenen Stadt bzw. Festung in ihrem Umfang und in ihrer sozialen Zusammensetzung stark voneinander unterscheiden konnten. Weitere Themen sind die Terminologie, die diplomatische Form und der Inhalt der Kapitulationsverträge (konkreter Zeitpunkt und Bedingungen der Übergabe, Garantien wie Eide und Geiseln, Abzugsbedingungen der Garnison, Schicksal der vorhandenen Mobilien und der Artillerie, Kriegsbeute, Kriegsgefangene, Regelung des »Seitenwechsels« der Betroffenen oder ggf. ihres Exils etc.). Ein eigener Abschnitt widmet sich der Vermittlungstätigkeit von Frauen.

Insgesamt gesehen kommt die Studie zu dem Ergebnis, der englische König Henry V. sei nicht nur als Eroberer aufgetreten, sondern auch darum bemüht gewesen, sich als legitimer Herrscher der Normandie zu präsentieren. Auf diese Weise habe er, parallel zum Einsatz militärischer Mittel und von Zwangsmaßnahmen, mit Hilfe von gut mit den französischen Verhältnissen vertrauten Verhandlungsführern und der Gewährung von Gnade, Verzeihung und Privilegien versucht, einen dauerhaften »Seitenwechsel« der Betroffenen zu erreichen. Innerhalb des Dialoges von Angreifern und Verteidigern ergebe sich jedoch ein deutlicher Verhaltensunterschied zwischen den Angehörigen des Militärs und den übrigen Einwohnern der Normandie, die sich mehrheitlich dafür entschieden, den Herrschaftswechsel zu akzeptieren, da ihnen andernfalls nur der Weg ins Exil geblieben wäre.

\section{Gisela Naegle, "Qui desiderat pacem, preparat bellum»}

[Hundertjähriger Krieg/Mittelalterliches Reich, 15. Jh.]

Der Traktat »Loquar in tribulacione« (1439/1440) von Jean Juvénal des Ursins (13881473), und der »Pentalogus« (1443) von Enea Silvio Piccolomini, dem zukünftigen Papst Pius II. (1405-1464), diskutieren die Frage von Krieg oder Frieden. Beide Autoren standen im Laufe ihres Lebens im Dienst des Königs und der Kirche. Ihre Argumente weisen einen engen Zusammenhang mit der politischen Aktualität der Ständeversammlungen und diplomatischen Verhandlungen der Zeit auf. In »Loquar in tribulacione« geht es um die Bewältigung des Hundertjährigen Krieges, im »Pentalogus« vor allem um die Italienpolitik des künftigen Kaisers Friedrich III. und die Wiederherstellung von Reichsrechten. 
Jean Juvénal des Ursins war entscheidend durch die engen Verbindungen seiner Familie zum Parlement geprägt. Die Untersuchung zeigt, dass seine Haltung zur Fortsetzung des Krieges gegen England zwiespältig war, aber durchweg von seiner tiefen Sorge um das Wohlergehen Frankreichs, des Königs und der Kirche bestimmt wurde. Eigene Erfahrungen mit Leid und Gefahren des Krieges in seiner Zeit als Bischof von Beauvais gaben schließlich den Ausschlag zugunsten des Friedens. Seine Argumentation weist Parallelen, aber auch deutliche Unterschiede zum »Pentalogus» auf, da für ihn der Zweck noch nicht alle Mittel heiligte und traditionelle Werte des mittelalterlichen Klerus im Vordergrund standen. Im Gegensatz dazu spielt im »Pentalogus « die Kunst der Diplomatie die Hauptrolle, zu deren integralen Bestandteilen Rhetorik, Taktik, List, Notwendigkeit, Nützlichkeitserwägungen und sogar Täuschungen gehören. Um erfolgreich zu sein, muss man Piccolomini zufolge die Schwächen der Gegner ausnutzen und versuchen, sie gegeneinander auszuspielen und zu entzweien. Er wünscht sich für Italien Frieden, empfiehlt als Mittel dazu jedoch den Krieg. Der Frieden ist für ihn nur eines von mehreren Zielen. Zusammenfassend lässt sich feststellen, dass im Zentrum des politischen Universums von Jean Juvénal des Ursins der bilaterale französisch-englische Konflikt stand, während das politische System Enea Silvio Piccolominis eine Vielzahl von Akteuren und unterschiedlichen Interessen der politischen Bühne der Zeit berücksichtigte. Beide Texte sprechen über die Ehre von König und Königreich, geben aber einen entgegengesetzten Rat: Friedrich III. soll den Krieg und Charles VII. den Frieden wählen.

\section{Jacques Paviot, Faire la paix pour faire la guerre}

\section{[Spätmittelalterliche Kreuzzüge]}

Kreuzzüge und Frieden waren unauflöslich miteinander verbunden, da der Friede unter den christlichen Fürsten als Voraussetzung für das Gelingen der Rückeroberung des Heiligen Landes angesehen wurde. Dies galt bereits für die Vorgeschichte des Ersten Kreuzzuges, die enge Verbindungen zu der im 10. Jh. von südfranzösischen Bischöfen lancierten Gottesfriedensbewegung aufwies. Die Kreuzzüge des 13. Jahrhunderts waren vor allem Unternehmungen kleiner Gruppen von Baronen und Individuen im Namen ihrer Herrscher. Der zunehmende Verlust der christlichen Kreuzfahrerstaaten regte im letzten Drittel des 13. Jahrhunderts eine Reihe von Autoren wie die Großmeister des Templer- und des Hospitaliterordens Jacques de Molay und Foulques de Villaret sowie Pierre Dubois und Roger de Stanegrave, einen englischen Hospitaliter, zum Verfassen von Projekten zu ihrer Rückgewinnung an. Ein Priester aus Lüttich namens Hugues schrieb den »Peregrinarius«, ein lateinisches Gedicht, das mit dem Ziel des Kampfes gegen die >Ungläubigen zum Frieden und zur Eintracht aufrief. In den beiden letzten Jahrzehnten des 14. Jahrhunderts wurden die Bemühungen um einen französisch-englischen Frieden und einen neuen Kreuzzug von Personen wie Philippe de Mézières, dem von den Mamelucken aus seinem Königreich vertriebenen König Leo von Kleinarmenien und Robert le Mennot (Robert l'Ermite) aufgenommen. Eustache Deschamps behandelte das Thema der Rückeroberung des Heiligen Landes in mehreren Balladen. Mitte des 15. Jahrhunderts bemühte sich Herzog Philipp der Gute (Philippe le Bon, 1396-1467) von Burgund, einen neuen Kreuzzug zustande zu bringen. Er schickte Gesandtschaften in verschiedene europäische Länder, die jedoch nur wenig Erfolg hatten. Angesichts der Unmöglichkeit, einen echten Frieden zu erreichen, spielten Prophezeiungen eine wichtige Rolle, die vor seinem Italienzug auf den französischen König Charles VIII. bezogen wurden. Während der 
Regierungszeit Louis' XIV. kam es noch einmal zu Projekten zur Zerschlagung des ottomanischen Reiches, die aber nicht in die Tat umgesetzt wurden.

\title{
Jean Devaux, »Entre le difficile et le possible»
}

\author{
[Burgund, 15. Jh.]
}

Der Abschluss des berühmten Friedens von Péronne am 14. Oktober 1468 zwischen dem burgundischen Herzog Charles le Téméraire (Karl der Kühne, 1433-1477) und dem französischen König Louis XI. (1423-1483) veranlasste den burgundischen Hofhistoriographen und Dichter George Chastelain († 1475) zur Abfassung eines theoretischen Traktats und eines dramatischen Werks. Üblicherweise werden beide Texte, das »Livre de Paix« und die moralité »La Paix de Péronne« als Ausdruck des tiefen Pessimismus ihres Autors gedeutet, der an der Aufrichtigkeit dieses Friedens und der Intentionen der beiden Fürsten gezweifelt habe. Im Gegensatz dazu wird hier die Auffassung vertreten, es handele sich um eine konstruktive Vorgehensweise. Das Ziel der beiden Werke bestehe darin, der Moral eine politische Funktion zuzuschreiben, die es erlaube, den Fürsten an seine Pflichten als Herrscher zu erinnern. Die lange Erfahrung Chastelains und die zahlreichen politischen Wechselfälle hätten zu einer erheblichen Desillusionierung des Autors geführt. Auf diese Weise sei er, besser als jeder andere, in der Lage gewesen, die Brisanz der politisch angespannten Lage zu erkennen, in der dieser Friedensschluss zustande kam. Dennoch habe ihn gerade diese besondere Hellsichtigkeit, in Verbindung mit seinem aufrichtigen Streben nach Frieden, zum Schreiben veranlasst. Entsprechend seiner Einsicht in die bevorstehenden Schwierigkeiten habe er seinem Herzog und dessen Umgebung die Mittel an die Hand geben wollen, die zu erwartenden Probleme zu meistern und einen dauerhaften Frieden zu sichern. Die beiden behandelten Werke zeigten daher im Gegensatz zu der üblicherweise vertretenen Interpretation gerade die Geschlossenheit und gedankliche Einheit des Werkes Chastelains und seinen unermüdlichen Einsatz für den Frieden und die Aussöhnung zwischen Frankreich und Burgund. Zugleich stellten sie eine vollendete Synthese des ein Jahrhundert lang im burgundischen Raum geführten Friedensdiskurses dar.

\section{Franck Collard, La royauté française et le renvoi de Marguerite d'Autriche}

[Frankreich, Ende 15. Jh.]

Das Spannungsverhältnis zwischen Krieg und Frieden war im Mittelalter Gegenstand philosophischer und juristischer Erörterungen, die Überlegungen zum Thema des gerechten Krieges miteinschlossen. Es handelte sich dabei aber auch um Fragen der Ideologie und der Kommunikation, da Friedensschlüsse und Kriegführung am Ende des Mittelalters nicht ohne einen Diskurs der Rechtfertigung oder der Glorifizierung auskamen, der die Ereignisse in einen allgemeineren biblischen und historischen Hintergrund einordnete. Der Beitrag untersucht diese Fragen anhand einer Fallstudie, die sowohl die französische als auch die deutsche Geschichte betrifft: die Zurückweisung der als Gemahlin des französischen Königs Charles VIII. (1403-1461) vorgesehenen Margarete von Österreich (1480-1530). Aus deutscher Sicht beging Charles VIII. durch die Eheschließung mit der bretonischen Erbtochter Anne de Bretagne (1477-1514) einen »Brautraub«, da sie bereits per Prokuration mit Margaretes Vater, Kaiser Maximilian I. (1459-1519), verheiratet war. Maximilian wurde durch diesen 
Vorgang also gleich doppelt, als Vater und Ehemann in seiner Ehre gekränkt. Von der französischen Seite wurde damals das Argument der Erhaltung des Friedens in einem Zusammenhang verwendet, in dem alles auf einen bevorstehenden Krieg hinwies.

Diese bekannte Episode der gemeinsamen Geschichte beider Länder wurde bereits häufiger unter ereignisgeschichtlichen, diplomatiegeschichtlichen oder affektiven Gesichtspunkten behandelt. Im Zentrum der Betrachtung steht hier jedoch der bisher weniger untersuchte Aspekt der Diskurs- und Ideologieschichte. Dabei liegt der Schwerpunkt der Studie auf der Argumentation der französischen Seite und dem »Schlagabtausch« zwischen dem zur Beschwichtigung nach Deutschland entsandten Pariser Humanisten und Kanonisten Robert Gaguin (1433-1501) und dem oberrheinischen Humanisten Jakob Wimpheling (1450-1528). Aus französischer Sicht spielte hier die Bezugnahme auf das Ideal des rex pacificus nach dem Vorbild des als Friedenskönig par excellence apostrophierten Saint Louis eine wichtige Rolle.

\section{Pierre Monnet, Conclusions}

In seiner Zusammenfassung der Ergebnisse der Tagung unterstreicht der Autor den prozesshaften Charakter von Verteidigung, diplomatischen Verhandlungen und Friedensschlüssen. Wie sich anhand zahlreicher Beiträge und der gewählten Sektionstitel zeige, seien Krieg und Frieden keine fest umrissenen, klar definierten oder streng voneinander abgrenzbaren Zustände. Ihr Verhältnis werde vielmehr durch zahlreiche Übergangsstadien und manchmal sogar recht widersprüchliche Kompromisse und "Zwischenstufen" gekennzeichnet. In diesem Zusammenhang erwiesen sich »kollektive Sicherheit«, »Legitimation/Delegitimation« und »Stabilisierung« als Schlüsselbegriffe der Untersuchungen. Eine Reihe von Beiträgen betone den Aspekt des aktiven Handelns, indem die Titel oder die inhaltliche Argumentation stark auf Verben wie »suchen« (rechercher), »retten« (sauver) oder »sichern« (assurer) zurückgriffen, die diese Vorgänge in ein komplexes Gefüge politischer und sozialer Aufbauprozesse einordneten, die nicht nur materielle Mittel, sondern auch und vor allem Personen und mit ihnen Diskurse und Rituale mobilisiert haben. $\mathrm{Zu}$ diesen Akteuren gehörten auch Städte und Ständeversammlungen. Im Zuge dieser Versammlungen wurde intensiv über Fragen des Krieges, des Friedens und deren Finanzierung diskutiert, so dass auf diese Weise eine »öffentliche« Auseinandersetzung mit diesen Themen stattfand, zu der Autoren theoretischer Traktate, Juristen und Theologen einen wichtigen Beitrag leisteten. Diese zusammenfassenden Betrachtungen werden durch eine Vorstellung neuerer Tendenzen und Themen der französischen und deutschen Historiographie und von Tagungen der letzten Jahre ergänzt. Am Ende steht der Ausblick auf interessante Erweiterungs- und Vertiefungsmöglichkeiten der Thematik wie der Rolle des Klerus und der Predigt, der Konzilien und der großen mittelalterlichen Friedenskonferenzen, der fiskalischen Aspekte von Verteidigung und Friedenssicherung oder der Erinnerungskultur von Krieg und Frieden. 